\title{
ASYMPTOTICALLY REGULAR OPERATORS IN GENERALIZED MORREY SPACES
}

\author{
SUN-SIG BYUN AND LUBOMIRA SOFTOVA
}

\begin{abstract}
We obtain Calderón-Zygmund type estimates in generalized Morrey spaces for nonlinear equations of $p$-Laplacian type. Our result is obtained under minimal regularity assumptions both on the operator and on the domain. This result allows us to study asymptotically regular operators. As a byproduct, we obtain also generalized Hölder regularity of the solutions under some minimal restrictions of the weight functions.
\end{abstract}

\section{INTRODUCTION}

In the present work we obtain global Calderón-Zygmund type estimates in generalized Morrey spaces $L^{q, \varphi}(\Omega)$ for operators of the kind

$$
\operatorname{div} \mathbf{a}(x, D u)=\operatorname{div}\left(|\mathbf{F}|^{p-2} \mathbf{F}\right) \quad \text { in } \Omega
$$

for some fixed $p \in(1, \infty)$ and bounded domain $\Omega$ with a rough boundary. Supposing first that $\mathbf{a}(x, \xi)$ is a regular elliptic operator of p-Laplacian type, we show, under minimal regularity assumptions, the implication

$$
|\mathbf{F}|^{p} \in L^{q, \varphi}(\Omega) \Longrightarrow|D u|^{p} \in L^{q, \varphi}(\Omega)
$$

for each $q \in[1, \infty)$ and measurable function $\varphi(x, r)$ satisfying suitable conditions. Recall that the operator $\mathbf{a}(x, \xi)$ is regular if it is differentiable and monotone with respect to $\xi \in \mathbb{R}^{n}$ for a.a. $x \in \mathbb{R}^{n}$. The implication (1.1) has been proved first by Calderón and Zygmund for the Poisson equation and in the framework of Lebesgue spaces. Since then, there have been a lot of works treating the validity of this implication for linear and nonlinear operators and in various function spaces, see for instance [2, 4, 5, 7, 8, 9, 10, 18, 23, and the references therein.

In 12. Chipot and Evans introduced the notion of asymptotically regular operators in the elliptic framework. Later, this notion has been extended to asymptotically regular operators with p-growth (see [25]). A local Calderón-Zygmund theory and partial Lipschitz regularity for asymptotically regular elliptic systems have been developed in [26, 27, while global results have been obtained in [15. These studies have been extended later to operators satisfying weaker asymptotic regularity condition of $p$-growth that allows to consider nonlinear problems oscillating around some regular problems (see [3]).

In the present work, we develop the Calderón-Zygmund theory for such operators in the settings of generalized Morrey spaces $L^{q, \varphi}$. For the description and some properties of these spaces, see for instance [8, 22] and the references therein. It is well known from the classical theory that if the gradient of a function belongs to a certain Morrey space, then the embedding theorems of Morrey and Campanato (cf. [11, 21] imply Hölder regularity of the function. In [13] the authors obtain an analogy of the classical Sobolev-Campanato embedding theorems but in the framework of generalized spaces. They prove sharp inclusion relations among generalized Morrey, Campanato and Hölder spaces, considering continuous weight function $\varphi(r): \mathbb{R}_{+} \rightarrow \mathbb{R}_{+}$, satisfying appropriate growth conditions. Restricting 
a class of weight functions, we show as a byproduct that the Calderón-Zygmund estimate implies generalized Hölder regularity of the same solution.

As mentioned above, we are going to study the Dirichlet problem for operators "close" to some regular operator under minimal regularity assumptions on the nonlinearity and the domain. For this goal, we consider domains with boundary satisfying the Reifenberg condition of flatness 24. This condition allows to the boundary to be very rough such that even the unit normal vector cannot be defined, but it should be flat enough such that it can be well approximated by $n-1$ dimensional planes. This structural condition implies the validity of the internal and external cone conditions and hence the validity of the fundamental results from the functional analysis. An example of such a boundary can be the Koch snowflake with an angle of the spike $\beta$ such that $0<\sin \beta<\delta<\frac{1}{8}$. A detailed overview of the properties of these domains can be found in [19, 29.

Throughout the paper, the letter $c$ will denote a universal constant that can be explicitly computed in terms of known quantities. The exact value of $c$ may vary from one occurrence to another. As usual, $D_{i} u=\frac{\partial u}{\partial x_{i}}, 1 \leq i \leq n$, and $D u=\left(D_{1} u, \ldots, D_{n} u\right)$ means the gradient vector field of $u$. For any bounded domain $\Omega \subset \mathbb{R}^{n}$, we denote by $|\Omega|$ the Lebesgue measure of $\Omega$ and by $d_{\Omega}$ the diameter of $\Omega$. Let $\mathcal{B}_{r}(y)$ be the ball centered in $y \in \Omega$ and of radius $r \in\left(0, d_{\Omega}\right]$, then $\Omega_{r}(y)=\Omega \cap \mathcal{B}_{r}(y)$. In addition the repeated-index summation convention is adopted.

\section{Regular $p$-LAPlace type PROBlems}

Let $\Omega \subset \mathbb{R}^{n}, n \geq 2$, be a bounded domain and $p \in(1, \infty)$ be fixed. We consider the following Dirichlet problem

$$
\begin{cases}\operatorname{div} \mathbf{a}(x, D u)=\operatorname{div}\left(|\mathbf{F}|^{p-2} \mathbf{F}\right) & \text { in } \Omega, \\ u(x)=0 & \text { on } \partial \Omega,\end{cases}
$$

where $\mathbf{F}=\left(f^{1}, \ldots, f^{n}\right) \in L^{p}\left(\Omega ; \mathbb{R}^{n}\right)$ is a given vector-valued function and $\mathbf{a}=$ $\mathbf{a}(x, \xi): \mathbb{R}^{n} \times \mathbb{R}^{n} \rightarrow \mathbb{R}^{n}$ is a Carathéodory map, i.e., measurable in $x$ for each $\xi$ and continuous in $\xi$ for a.a. $x \in \Omega$.

Definition 2.1. The operator $\mathbf{a}(x, \xi)$ is regular if it is differentiable in $\xi$ and satisfies the following structural conditions

$$
\left\{\begin{array}{l}
\gamma|\xi|^{p-2}|\eta|^{2} \leq\left\langle D_{\xi} \mathbf{a}(x, \xi) \eta, \eta\right\rangle \\
|\mathbf{a}(x, \xi)|+|\xi|\left|D_{\xi} \mathbf{a}(x, \xi)\right| \leq \Lambda|\xi|^{p-1}
\end{array}\right.
$$

for each $0 \neq \xi, \eta \in \mathbb{R}^{n}$, for a.a. $x \in \mathbb{R}^{n}$, and for some positive constants $\gamma$ and $\Lambda$.

The condition (2.2) easily implies monotonicity of a, i.e.,

$$
\begin{aligned}
& \langle\mathbf{a}(x, \xi)-\mathbf{a}(x, \eta), \xi-\eta\rangle \\
& \quad \geq \gamma_{1} \begin{cases}|\xi-\eta|^{p} & \text { if } p \geq 2 \\
|\xi-\eta|^{2}(1+|\xi|+|\eta|)^{p-2} & \text { if } 1<p<2,\end{cases}
\end{aligned}
$$

where $\gamma_{1}$ depends only on $\gamma, n$ and $p$.

Recall that a weak solution of (2.1) means a function $u \in W_{0}^{1, p}(\Omega), p \in(1, \infty)$ that satisfies

$$
\int_{\Omega}\langle\mathbf{a}(x, D u), D \phi\rangle d x=\int_{\Omega}\left\langle|\mathbf{F}|^{p-2} \mathbf{F}, D \phi\right\rangle d x
$$

for any $\phi \in W_{0}^{1, p}(\Omega)$. 
The unique weak solvability of (2.1) follows by the Minty-Browder method in $L^{p}$ that gives the estimate

$$
\|D u\|_{L^{p}(\Omega)} \leq c\|\mathbf{F}\|_{L^{p}(\Omega)},
$$

where the constant $c$ depends only on $n, p, \gamma, \lambda$ and $|\Omega|$, see for instance [1, 14.

We suppose that the dependence of $x$ in the nonlinear term a is of small $B M O$ (bounded mean oscillation) type. In order to describe this regularity, we have to be able to measure the oscillation of the mapping $x \rightarrow \frac{\mathbf{a}(x, \xi)}{|\xi|^{p-1}}$ over balls, uniformly in $\xi \in \mathbb{R}^{n} \backslash\{0\}$. To this end, we introduce the function

$$
\theta(\mathbf{a} ; r, y)(x)=\sup _{\xi \in \mathbb{R}^{n} \backslash\{0\}} \frac{\left|\mathbf{a}(x, \xi)-\overline{\mathbf{a}}_{\mathcal{B}_{r}(y)}(\xi)\right|}{|\xi|^{p-1}},
$$

where $\overline{\mathbf{a}}_{\mathcal{B}_{r}(y)}(\xi)=\frac{1}{\left|\mathcal{B}_{r}(y)\right|} \int_{\mathcal{B}_{r}(y)} \mathbf{a}(x, \xi) d x$ is the integral average of a over $\mathcal{B}_{r}(y)$ with respect to $x$ for any fixed $\xi \in \mathbb{R}^{n} \backslash\{0\}$.

Definition 2.2. We say that the vector field a is $(\delta, R)$-vanishingif

$$
\sup _{0<r \leq R} \sup _{y \in \mathbb{R}^{n}} f_{\mathcal{B}_{r}(y)} \theta(\mathbf{a} ; r, y)(x) d x \leq \delta .
$$

As it concerns the domain $\Omega$, we impose the following kind of flatness.

Definition 2.3. We say that $\Omega$ is $(\delta, R)$-Reifenberg flat if for every $x_{0} \in \partial \Omega$ and every $r \in(0, R]$, there exists a coordinate system $\left\{y_{1}, \ldots, y_{n}\right\}$ centered in $x_{0}$, which can depend on $r$ and $x_{0}$, so that $x_{0}=0$ with respect to it and

$$
\mathcal{B}_{r}(0) \cap\left\{y_{n}>\delta r\right\} \subset B_{r}(0) \cap \Omega \subset B_{r}(0) \cap\left\{y_{n}>-\delta r\right\} .
$$

The problem (2.1) is invariant under scaling and normalization, as it can be seen by the following lemma (see [7, Lemma 2.5]).

Lemma 2.4. For each $\lambda \geq 1$ and $0<\rho \leq 1$, we define the rescaled maps:

Then

$$
\begin{aligned}
& \tilde{\mathbf{a}}(x, \xi)=\frac{\mathbf{a}(\rho x, \lambda \xi)}{\lambda^{p-1}}, \quad \tilde{\Omega}=\left\{\frac{x}{\rho}: x \in \Omega\right\}, \\
& \tilde{u}(x)=\frac{u(\rho x)}{\lambda x}, \quad \tilde{\mathbf{F}}(x)=\frac{\mathbf{F}(\rho x)}{\lambda} .
\end{aligned}
$$

(1) $\tilde{u} \in W_{0}^{1, p}(\tilde{\Omega})$ is the weak solution of

$$
\operatorname{div} \tilde{\mathbf{a}}(x, D \tilde{u})=\operatorname{div}\left(|\tilde{\mathbf{F}}|^{p-2} \tilde{\mathbf{F}}\right) \quad \text { in } \tilde{\Omega} .
$$

(2) ã satisfies the structural conditions (2.2) with the same constants $\Lambda, \gamma$.

(3) $\tilde{\mathbf{a}}$ is $\left(\delta, \frac{R}{\rho}\right)$-vanishing.

(4) $\tilde{\Omega}$ is $\left(\delta, \frac{R}{\rho}\right)$-Reifenberg flat.

Note that thanks to the scaling invariance property, one can take for simplicity $R=1$ or any other constant bigger than or equal to 1 . On the other hand, $\delta$ is a small positive constant, say $0<\delta<1 / 8$, being invariant under such a scaling argument.

We suppose that $|\mathbf{F}|^{p} \in L^{q, \varphi}(\Omega)$ with $q \in(1, \infty)$ and $\varphi$ satisfying (3.1)-(3.3), as described in Section 3 below, which implies that $|\mathbf{F}|^{p} \in L^{1}(\Omega)$. In fact,

$$
\begin{aligned}
\left\||\mathbf{F}|^{p}\right\|_{L^{q}(\Omega)}^{q} & =\varphi\left(y, r^{*}\right) \frac{1}{\varphi\left(y, r^{*}\right)} \int_{\Omega}|\mathbf{F}(x)|^{p q} d x \\
& \leq \varphi(0, d)\left\||\mathbf{F}|^{p}\right\|_{L^{q, \varphi}(\Omega)}^{q} .
\end{aligned}
$$

Then the Hölder inequality implies

$$
\left\||\mathbf{F}|^{p}\right\|_{L^{1}(\Omega)} \leq|\Omega|^{1-\frac{1}{q}}\left\||\mathbf{F}|^{p}\right\|_{L^{q}(\Omega)} \leq c\left\||\mathbf{F}|^{p}\right\|_{L^{q, \varphi}(\Omega)},
$$


which ensures the existence of a unique weak solution $u \in W_{0}^{1, p}(\Omega)$ of (2.1), where the constant $c$ depends on $n, p, \varphi,|\Omega|$. Moreover, it is shown in [7] that this solution belongs to $W_{0}^{1, q}(\Omega)$ and we have the following estimate

$$
\left\||D u|^{p}\right\|_{L^{q}(\Omega)} \leq c\left\||\mathbf{F}|^{p}\right\|_{L^{q}(\Omega)} .
$$

Our goal is to develop the Calderón-Zygmund theory for the problem (2.1) in the setting of the generalized Morrey spaces. Namely, taking $|\mathbf{F}|^{p} \in L^{q, \varphi}(\Omega)$ with $q \in(1, \infty)$ and $\varphi$ satisfying suitable doubling and integral conditions, we are going to show that the gradient $|D u|^{p}$ belongs to the same space $L^{q, \varphi}(\Omega)$ with the desired estimate (3.5) below, which is a correct and natural extension of (2.9) in Lebesque spaces to the one in generalized Morrey spaces.

\section{Generalized Morrey type Regularity}

Let $\varphi=\varphi(y, r): \mathbb{R}^{n} \times \mathbb{R}_{+} \rightarrow \mathbb{R}_{+}$be a measurable function and $1<q<\infty$. The generalized Morrey space $L^{q, \varphi}(\Omega)$ consists of all measurable functions $f$ for which the following norm is finite:

$$
\|f\|_{L^{q, \varphi}(\Omega)}=\sup _{\substack{y \in \Omega \\ 0<r \leq d_{\Omega}}}\left(\frac{1}{\varphi(y, r)} \int_{\Omega_{r}(y)}|f(x)|^{q} d x\right)^{\frac{1}{q}}
$$

where $\varphi(y, r)=\varphi\left(\mathcal{B}_{r}(y)\right)$ depends on the ball.

We assume that for any fixed $y, z \in \mathbb{R}^{n}$ and $r, s>0$, there are positive constants $\varkappa_{1}, \varkappa_{2}$ and $\varkappa_{3}$, independent of $y, z, r$ and $s$, such that

$$
\begin{aligned}
& \varkappa_{1} \leq \frac{\varphi(y, s)}{\varphi(y, r)} \leq \varkappa_{2}, \quad r \leq s \leq 2 r, \varkappa_{1}, \varkappa_{2}>0, \\
& \int_{r}^{\infty} \frac{\varphi(y, t)}{t^{n+1}} d t \leq \varkappa_{3} \frac{\varphi(y, r)}{r^{n}}, \quad \varkappa_{3} \geq 1 \\
& \varphi(y, r) \leq \varphi(z, s) \quad \text { for } \quad \mathcal{B}_{r}(y) \subset \mathcal{B}_{s}(z) .
\end{aligned}
$$

Since $\Omega$ is bounded, for any $y \in \Omega$, it holds $\sup _{z \in \Omega}|y-z| \leq d_{\Omega}$. Hence there exists $r^{*} \leq d_{\Omega}$ such that $\Omega \subset \mathcal{B}_{r^{*}}(y) \subset \mathcal{B}_{d}(0)$ for some $d \geq d_{\Omega}$. Then (3.3) gives that for all $y \in \Omega$,

$$
\varphi\left(y, r^{*}\right) \leq \varphi(0, d) .
$$

Moreover, the monotonicity condition (3.3) implies (cf. [8])

$$
\sup _{\substack{y \in \Omega \\ r>0}} \frac{\left|\Omega_{r}(y)\right|}{\varphi(y, r)}<\varkappa_{4}
$$

which is equivalent to $\left\|\chi_{\Omega}\right\|_{L^{q, \varphi}(\Omega)}<\varkappa_{4}$, where $\chi_{\Omega}$ is the characteristic function of $\Omega$.

Theorem 3.1. Let $q \in[1, \infty)$ and $\varphi: \mathbb{R}^{n} \times \mathbb{R}_{+} \rightarrow \mathbb{R}_{+}$be a weight satisfying (3.1)(3.3). Assume that $\mathbf{a}(x, \xi)$ is regular and $|\mathbf{F}|^{p} \in L^{q, \varphi}(\Omega)$. Then there exists a small positive constant $\delta_{0}=\delta_{0}(n, p, q, \gamma, L, \varphi)$ such that if $\mathbf{a}$ and $\Omega$ satisfy (2.6) and (2.7), respectively with $\delta_{0}$, then $|D u|^{p} \in L^{q, \varphi}(\Omega)$ and we have the following estimate

$$
\left\||D u|^{p}\right\|_{L^{q, \varphi}(\Omega)} \leq c|||\mathbf{F}|^{p} \|_{L^{q, \varphi}(\Omega)}
$$

with constant $c$ depending on known quantities.

Proof. In [7, the authors study the weak solvability of (2.1) in the weighted Lebesgue spaces $L_{w}^{q}(\Omega)$ with $w$ a Muckenhoupt weight. Recall that $w$ belongs to the Muckenhoupt class $A_{q}, q \in(1, \infty)$, if

$$
[w]_{q}:=\sup _{\mathcal{B}}\left(\frac{1}{|\mathcal{B}|} \int_{\mathcal{B}} w(x) d x\right)\left(\frac{1}{|\mathcal{B}|} \int_{\mathcal{B}} w(x)^{-\frac{1}{q-1}} d x\right)^{q-1}<+\infty
$$


where the supremum is taken over all balls $\mathcal{B} \subset \mathbb{R}^{n}$. If $q=1$ we say that $w \in A_{1}$ if

$$
\frac{1}{|\mathcal{B}|} \int_{\mathcal{B}} w(x) d x \leq[A]_{1} \underset{\mathcal{B}}{\operatorname{essinf}} w(x)
$$

for some positive constant $[A]_{1}$. By [7], we have that if $|\mathbf{F}|^{p} \in L_{w}^{q}(\Omega)$ with $w \in A_{q}$, then $|D u|^{p} \in L_{w}^{q}(\Omega)$ and verifies the estimate

$$
\int_{\Omega}|D u|^{p q} w(x) d x \leq c \int_{\Omega}|\mathbf{F}(x)|^{p q} w(x) d x .
$$

For any Borel set B, the Coifman-Rochberg result (cf. 28]) asserts that the maximal operator of the characteristic function of $B$

$$
\mathcal{M} \chi_{B}(x)=\sup _{x \in \mathcal{B}} \frac{1}{|\mathcal{B}|} \int_{\mathcal{B}} \chi_{B}(z) d z
$$

verifies that $\left(\mathcal{M} \chi_{B}\right)^{\sigma} \in A_{1}$ for any $\sigma \in(0,1)$. Because of the increasing property of the $A_{q}$ classes, i.e., if $w \in A_{q}$, then $w \in A_{p}$ whenever $p>q$, we have that $\left(\mathcal{M} \chi_{B}\right)^{\sigma} \in A_{q}$ for each $q>1$.

Let us extend $u$ and $\mathbf{F}$ as zero outside $\Omega$ and recall that the assumptions on the regularity of $\partial \Omega$ allow us to do it. Let $y \in \Omega$ and $r>0$. Then we calculate:

$$
\begin{aligned}
I & :=\frac{1}{\varphi(y, r)} \int_{\Omega_{r}(y)}|D u(x)|^{p q} d x \\
& =\frac{1}{\varphi(y, r)} \int_{\mathbb{R}^{n}}|D u(x)|^{p q} \chi_{\Omega_{r}(y)}(x) d x \\
& \leq \frac{1}{\varphi(y, r)} \int_{\mathbb{R}^{n}}|D u(x)|^{p q} \chi_{\mathcal{B}_{r}(y)}(x) d x \\
& \leq \frac{1}{\varphi(y, r)} \int_{\mathbb{R}^{n}}|D u(x)|^{p q}\left(\mathcal{M}_{\left.\chi_{\mathcal{B}_{r}(y)}(x)\right)^{\sigma} d x}\right. \\
& \leq \frac{c}{\varphi(y, r)} \int_{\mathbb{R}^{n}}|\mathbf{F}(x)|^{p q}\left(\mathcal{M}_{\left.\chi_{\mathcal{B}_{r}(y)}(x)\right)^{\sigma} d x,}\right.
\end{aligned}
$$

where we have used the fact that

$$
\chi_{\mathcal{B}_{r}(y)}(x) \leq \mathcal{M} \chi_{\mathcal{B}_{r}(y)}(x)=\sup _{x \in \mathcal{B}_{\rho}} \frac{\left|\mathcal{B}_{r}(y) \cap \mathcal{B}_{\rho}\right|}{\left|\mathcal{B}_{\rho}\right|} \leq\left(\mathcal{M}_{\chi_{\mathcal{B}_{r}(y)}}\right)^{\sigma}(x) \leq 1
$$

and the estimate (3.8).

Simplifying the notations by writing $\mathcal{B}=\mathcal{B}_{r}(y)$ and $2 \mathcal{B}=\mathcal{B}_{2 r}(y)$, we write the dyadic decomposition of $\mathbb{R}^{n}$ related to $\mathcal{B}$ as

$$
\mathbb{R}^{n}=2 \mathcal{B} \cup\left(\bigcup_{k=1}^{\infty} 2^{k+1} \mathcal{B} \backslash 2^{k} \mathcal{B}\right)
$$

Then (3.9) becomes

$$
\begin{aligned}
I \leq & \frac{c}{\varphi(y, r)} \int_{2 \mathcal{B}}|\mathbf{F}(x)|^{p q}\left(\mathcal{M} \chi_{\mathcal{B}}(x)\right)^{\sigma} d x \\
& +c \sum_{k=1}^{\infty} \frac{1}{\varphi(y, r)} \int_{2^{k+1} \mathcal{B} \backslash 2^{k} \mathcal{B}} \frac{1}{\varphi(y, r)}|\mathbf{F}(x)|^{p q}\left(\mathcal{M} \chi_{\mathcal{B}}(x)\right)^{\sigma} d x \\
= & : c I_{0}+c \sum_{k=1}^{\infty} I_{k} .
\end{aligned}
$$

Let us estimate the maximal function in the above integrals:

○ If $x \in 2 \mathcal{B}$, then the supremum in 3.10 is attained when $\mathcal{B} \subseteq \mathcal{B}_{\rho}$, and whence $\mathcal{M}_{\chi_{\mathcal{B}}}(x) \leq 1$. 
○ If $x \in 2^{k+1} \mathcal{B} \backslash 2^{k} \mathcal{B}$, then

$$
\frac{r^{n}}{\left(2^{k+1} r+r\right)^{n}} \leq \mathcal{M} \chi_{\mathcal{B}}(x) \leq \frac{r^{n}}{\left(2^{k} r+r\right)^{n}},
$$

which permits to compare $\mathcal{M} \chi_{\mathcal{B}}(x) \sim 2^{-k n}$.

Then making use of (3.1), we can estimate (3.11) in the following way:

$$
\begin{aligned}
I_{0} & \leq \frac{c}{\varphi(y, r)} \int_{2 \mathcal{B}}|\mathbf{F}(x)|^{p q} d x \\
& \leq \frac{c \varkappa_{2}}{\varphi(y, 2 r)} \int_{2 \mathcal{B}}|\mathbf{F}(x)|^{p q} d x \leq c\left\||\mathbf{F}|^{p}\right\|_{L^{q, \varphi}(\Omega)}^{q}, \\
I_{k} & \leq \frac{c}{\varphi(r, y)} \int_{2^{k+1} \mathcal{B} \backslash 2^{k} \mathcal{B}}|\mathbf{F}(x)|^{p q} \frac{1}{2^{k n \sigma}} d x \\
& \leq \frac{c \varkappa_{2}^{k+1}}{2^{k n \sigma}} \frac{1}{\varphi\left(y, 2^{k+1} r\right)} \int_{2^{k+1} \mathcal{B}}|\mathbf{F}(x)|^{p q} d x \\
& \leq c \varkappa_{2} \frac{\varkappa_{2}^{k}}{2^{n \sigma k}}\left\||\mathbf{F}|^{p}\right\|_{L^{q, \varphi}(\Omega)}^{q} .
\end{aligned}
$$

Choosing $\sigma \in\left(\frac{\log _{2} \varkappa_{2}}{n}<\sigma<1\right)$ and summing up the integrals $I_{k}$ we discover

$$
I \leq c \sum_{k=0}^{\infty}\left(\frac{\varkappa_{2}}{2^{\sigma n}}\right)^{k}\left\||\mathbf{F}|^{p}\right\|_{L^{q, \varphi}(\Omega)}^{q} \leq c\left\||\mathbf{F}|^{p}\right\|_{L^{q, \varphi}(\Omega)}^{q},
$$

where the constant depends on $n, p, q$, and $|\Omega|$.

Finally combining (3.9) and (3.14) and taking the supremum with respect to all balls $\mathcal{B}$, we obtain the desired estimate (3.5).

\section{Generalized Hölder Regularity}

If we restrict the class of weight functions, then we can obtain, through the results of 13, a generalized Hölder regularity of the solution $u$ to the problem (2.1). Suppose that $\varphi(r): \mathbb{R}_{+} \rightarrow \mathbb{R}_{+}$is continuous. The generalized Hölder space $C^{0, \varphi}(\Omega)$ consists of all continuous functions $\varphi(r): \mathbb{R}_{+} \rightarrow \mathbb{R}_{+}$for which the following seminorm

$$
[f]_{C^{0, \varphi}(\Omega)}=\sup _{\substack{x, y \in \Omega \\ x \neq y}} \frac{|f(x)-f(y)|}{\varphi(|x-y|)}
$$

is finite. Obviously, if $\lim _{r \rightarrow 0_{+}} \varphi(r)=0$ then $C^{0, \varphi}(\Omega)$ concontains uniformly continuous functions. Using the technique of the rearrangement invariant (r.i.) spaces, Cianchi and Pick obtained a correct condition on the weight function for the embedding of the Sobolev spaces into generalized Hölder spaces. Precisely, the [13, Theorem 1.3] asserts that if $X(\Omega)$ is r.i. space and $\varphi$ is a strictly positive continuous function on $(0, \infty)$, then the following assertions are equivalent:

(i) A positive constant $c$ exists such that

$$
\|u\|_{C^{0, \varphi}(Q)} \leq c\|D u\|_{X(Q)}
$$

for every $u \in W^{1} X(Q)$;

(ii) $\sup _{0<\rho<|Q|} \frac{1}{\varphi\left(\rho^{\frac{1}{n}}\right)}\left\|t^{-\frac{1}{n^{\prime}}} \chi_{(0, \rho)}(t)\right\|_{\bar{X}^{\prime}(0,|Q|)}<\infty$.

Recall that if $X(Q)$ is r.i. space, then $\bar{X}(0,|Q|)$ is its representation space, while $X^{\prime}(Q)$ and $\bar{X}^{\prime}(0,|Q|)$ are the topological dual spaces, respectively of $X(Q)$ and $\bar{X}(0,|Q|)$ and $Q$ is a cube in $\mathbb{R}^{n}$. Without loss of generality, we can take $Q$ such that $\Omega \subset Q$ and $|Q|=d_{\Omega}^{n}$. In order to apply [13, Theorem 1.3], we extend $u$ 
and $\mathbf{F}$ as zero outside $\Omega$, take $X(Q)=L^{p q}(Q)$ and hence $\bar{X}(0,|Q|)=L^{p q}\left(0, d_{\Omega}^{n}\right)$, $\bar{X}^{\prime}=(0,|Q|)=L^{\frac{p q}{p q-1}}\left(0, d_{\Omega}^{n}\right)$. Then the condition (ii) becomes

$$
\sup _{0<\rho<d_{\Omega}^{n}} \frac{1}{\varphi\left(\rho^{1 / n}\right)}\left[\int_{0}^{\rho}\left(t^{-\frac{1}{n^{\prime}}}\right)^{\frac{p q}{p q-1}} d t\right]^{\frac{p q-1}{p q}}<\infty,
$$

where $\frac{1}{n^{\prime}}=1-\frac{1}{n}$. Direct calculations and change of the variables $r=\rho^{\frac{1}{n}}$ give that for any fixed $n$ and $p$ the condition (ii) becomes

$$
\sup _{0<r<d_{\Omega}} \frac{r^{1-\frac{n}{p q}}}{\varphi(r)}<\infty, \quad \forall q>\max \left\{1, \frac{n}{p}\right\} .
$$

Then if $\varphi(r)$ satisfies (4.1), then it holds

$$
\|u\|_{C^{0, \varphi}(\Omega)} \leq c\|D u\|_{L^{p q}(\Omega)} \leq c\|\mathbf{F}\|_{L^{p q}(\Omega)} .
$$

\section{Asymptotically REgular Problems}

Consider now the following nonlinear elliptic problem

$$
\begin{cases}\operatorname{div} \mathbf{b}(x, D u)=\operatorname{div}\left(|\mathbf{F}|^{p-2} \mathbf{F}\right) & \text { in } \Omega \\ u=0 & \text { on } \partial \Omega,\end{cases}
$$

where $\mathbf{F} \in L^{p, \varphi}\left(\Omega ; \mathbb{R}^{n}\right)$ is a given vector-valued function with $p \in(1, \infty)$ and $\varphi(x, r)$ satisfying (3.1)-(3.3).

Definition 5.1. Let $\mathbf{a}(x, \xi)$ be a regular operator in the sense of the Definition [2.1. The operator $\mathbf{b}(x, \xi): \mathbb{R}^{n} \times \mathbb{R}^{n} \rightarrow \mathbb{R}^{n}$, satisfying the Carathéodory conditions, is asymptotically $\delta$-regular with $\mathbf{a}(x, \xi)$ if

$$
\limsup _{|\xi| \rightarrow \infty} \frac{|\mathbf{b}(x, \xi)-\mathbf{a}(x, \xi)|}{|\xi|^{p-1}} \leq \delta,
$$

uniformly with respect to $x \in \mathbb{R}^{n}$.

Let us note that the condition (5.2) implies that

$$
|\mathbf{b}(x, \xi)-\mathbf{a}(x, \xi)| \leq \omega(|\xi|)\left(1+|\xi|^{p-1}\right) \text { and } \limsup _{r \rightarrow \infty} \omega(r) \leq \delta,
$$

where $\omega: \mathbb{R}^{+} \rightarrow \mathbb{R}^{+}$is a uniformly bounded function, defined as

$$
\omega(|\xi|)=\sup _{x \in \mathbb{R}^{n}} \frac{|\mathbf{b}(x, \xi)-\mathbf{a}(x, \xi)|}{|\xi|^{p-1}} .
$$

The notion of asymptotic regularity has been introduced in [12] assuming that $\lim _{r \rightarrow \infty} \omega(r)=0$. Later, this condition have been relaxed in [3] taking boundedness of $\omega(r)$ on infinity, including such a way oscillating operators with small oscillation with respect to $\xi$, as for example,

$$
\mathbf{b}(x, \xi)=\mathbf{a}(x, \xi)+\delta \sin \left(|\xi|^{2}\right)|\xi|^{p-2 \xi}
$$

where $\mathbf{a}(x, \xi)$ is regular. In order to obtain Calderón-Zygmund type estimates for the problem (5.1), we need to transform it in a suitable regular problem for which we can apply the results obtained in Section 3. Let us introduce the operator

$$
\mathbf{c}(x, \xi)=\frac{\mathbf{b}(x, \xi)-\mathbf{a}(x, \xi)}{|\xi|^{p-1}}, \quad|\xi| \neq 0 .
$$

Obviously, it is a Carathéodory map. Moreover, $\lim \sup _{|\xi| \rightarrow \infty} \mathbf{c}(x, \xi) \leq \delta$, hence there exists $M(\delta, \omega)>1$ such that $|\mathbf{c}(x, \xi)| \leq 2 \delta$ if $|\xi| \geq M$, uniformly with respect to $x \in \Omega$. Since $\xi \rightarrow \mathbf{c}(x, \xi)$ is continuous for each fixed $x$ over the sphere $|\xi|=M$, 
we can apply the classical theory [16] to construct a harmonic function in the ball $\mathcal{B}_{M}(0)=\mathcal{B}_{M}$. Taking the Poisson kernel

$$
\mathcal{P}(\xi, \eta)=\frac{M^{2}-|\xi|^{2}}{M \omega_{n-1}|\xi-\eta|^{n}} \quad\left(\xi \in \mathcal{B}_{M}, \eta \in \partial \mathcal{B}_{M}\right),
$$

we consider the Poisson integral

$$
P[\mathbf{c}(x, \cdot)](\xi)=\int_{\partial \mathcal{B}_{M}} \mathbf{c}(x, \eta) \mathcal{P}(\xi, \eta) d \sigma_{\eta} \quad\left(\xi \in \mathcal{B}_{M}\right) .
$$

Then $\xi \rightarrow P[\mathbf{c}(x, \cdot)](\xi)$ is harmonic function in $\mathcal{B}_{M}$ with respect to $\xi$ and coincides with $\mathbf{c}(x, \xi)$ on $\partial \mathcal{B}_{M}$. Define the function

$$
\tilde{\mathbf{c}}(x, \xi):= \begin{cases}\mathbf{c}(x, \xi) & \text { if }|\xi| \geq M \\ P[\mathbf{c}(x, \cdot)](\xi) & \text { if }|\xi|<M\end{cases}
$$

which is a Carathéodory map in $\mathbb{R}^{n} \times \mathbb{R}^{n}$ such that $|\tilde{\mathbf{c}}(x, \xi)| \leq 2 \delta$ for each $\xi \in \mathbb{R}^{n}$ by the maximum principle.

Lemma 5.2. Let $u \in W_{0}^{1, p}(\Omega)$ be a weak solution of the asymptotical problem (5.1). Then by [3], it is a weak solution of the problem

$$
\begin{cases}\operatorname{div} \tilde{\mathbf{c}}(x, D u)=\operatorname{div}\left(|\mathbf{G}|^{p-2} \mathbf{G}\right) & \text { in } \Omega \\ u=0 & \text { on } \partial \Omega,\end{cases}
$$

where $\mathbf{G}$ is defined by

$$
\mathbf{G}=\frac{|\mathbf{F}|^{p-2} \mathbf{F}+|D u|^{p-1} \chi_{\{|D u|<M\}}(\tilde{\mathbf{c}}(x, D u))-\mathbf{c}(x, D u)}{\left.|| \mathbf{F}\right|^{p-2} \mathbf{F}+|D u|^{p-1} \chi_{\{|D u|<M\}}(\tilde{\mathbf{c}}(x, D u))-\left.\mathbf{c}(x, D u)\right|^{\frac{p-2}{p-1}}}
$$

if the denominator is different from zero, and $\mathbf{G}=0$ when the denominator vanishes. Hence $\mathbf{G} \in L^{p}\left(\Omega ; \mathbb{R}^{n}\right)$ and

$$
\|\mathbf{G}\|_{L^{p}(\Omega)} \leq C\left(\|\mathbf{F}\|_{L^{p}(\Omega)}+1\right) .
$$

Since $|\mathbf{F}|^{p} \in L^{q, \varphi}(\Omega)$ for some $q \in[1, \infty)$ and any fixed $p \in(1, \infty), \mathbf{F} \in L^{p q, \varphi}\left(\Omega ; \mathbb{R}^{n}\right)$. We are going to show that $\mathbf{G} \in L^{p q, \varphi}\left(\Omega ; \mathbb{R}^{n}\right)$.

Proof. For $\delta$ small, it holds $|\tilde{\mathbf{c}}(x, D u)| \leq 2 \delta<2$ uniformly with respect to $x$. Then, as in [3],

$$
\begin{aligned}
& \left.|| D u\right|^{p-1} \mathbf{c}(x, D u)|=| \mathbf{b}(x, D u)-\mathbf{a}(x, D u) \mid \\
& \quad \leq \omega(|D u|)\left(1+|D u|^{p-1}\right) \leq\|\omega\|_{\infty}\left(1+|D u|^{p-1}\right)
\end{aligned}
$$

and hence

$$
\left.|| D u\right|^{p-1} \chi_{\{|D u|<M\}} \mathbf{c}(x, D u) \mid \leq 2\|\omega\|_{\infty} M^{p-1} .
$$

Accordingly, $|\mathbf{G}|^{p} \leq c\left(|\mathbf{F}|^{p}+\left(1+\|\omega\|_{\infty}\right)^{\frac{p}{p-1}} M^{p}\right)$. Then

$$
|\mathbf{G}|^{p q} \leq c\left(|\mathbf{F}|^{p q}+\left(1+\|\omega\|_{\infty}\right)^{\frac{p q}{p-1}} M^{p q}\right)
$$

with a constant $c=c(p, q)$. Applying (3.4), we get

$$
\begin{aligned}
\frac{1}{\varphi(y, r)} \int_{\Omega_{r}}|\mathbf{G}(x)|^{p q} d x & \leq\left.\frac{c}{\varphi(y, r)} \int_{\Omega_{r}} \mathbf{F}(x)\right|^{p q} d x+c \frac{\left|\Omega_{r}\right|}{\varphi(y, r)} \\
& \leq c\left(\|\mathbf{F}\|_{L^{p q, \varphi}}^{p q}+1\right)
\end{aligned}
$$

with a constant depending on known quantities and $\|\omega\|_{\infty}$. Taking the supremum over $y \in \Omega$ and $r \in(0, \operatorname{diam} \Omega]$, we get

$$
\|\mathbf{G}\|_{L^{p q, \varphi}(\Omega)}^{p q} \leq\left(\|\mathbf{F}\|_{L^{p q, \varphi}}^{p q}+1\right) .
$$


The desired regularity requirements of the operator $\tilde{\mathbf{c}}$ follows by the next lemma that is proved in [3].

Lemma 5.3. Assume that $\mathbf{b}(x, \xi)$ is asymptotically $\delta$-regular with $\mathbf{a}(x, \xi)$. Then

(1) $\tilde{\mathbf{c}}(x, \xi)$ is regular if $0<\delta<\min \left\{\frac{\Lambda}{4 n(|p-2|+1)}, 1\right\}$.

(2) $\tilde{\mathbf{c}}(x, \xi)$ is $(5 \delta, R)$-vanishing.

We are now ready to state and prove our desired Calderón-Zygmund theory with the desired estimate for the asymptotically regular problem (5.1) in generalized Morrey spaces.

Theorem 5.4. For any $q \in[1, \infty)$ and $\varphi$ satisfying (3.1)-(3.3), assume $|\mathbf{F}|^{p} \in$ $L^{q, \varphi}\left(\Omega ; \mathbb{R}^{n}\right)$. Then there exists $\delta=\delta(n, p, q, \gamma, L, \varphi)>0$ such that if $\mathbf{b}(x, \xi)$ is asymptotically $\delta$-regular with $\mathbf{a}(x, \xi)$ satisfying and (2.6) and if $\Omega$ satisfies (2.7), then any weak solution $u \in W_{0}^{1, p}(\Omega)$ of (5.1) verifies

$$
\left\||D u|^{p}\right\|_{L^{q, \varphi}(\Omega)} \leq c\left(\left\||\mathbf{F}|^{p}\right\|_{L^{q, \varphi}(\Omega)}+1\right)
$$

for some positive constant $c=c(n, p, q, \gamma, L, \varphi,|\Omega|)$.

Proof. Let $\delta_{0}$ be the constant from Theorem 3.1 and let

$$
\delta_{1}=\min \left\{\frac{\lambda}{4 n(|p-2|+1)}, 1\right\} .
$$

We set $\delta=\frac{1}{5} \min \left\{\delta_{0}, \delta_{1}\right\}>0$. Let $u \in W_{0}^{1, p}(\Omega)$ be a weak solution of (5.1). Since $\mathbf{a}$ is $\left(\delta_{0}, R\right)$-vanishing, it follows from Lemma 5.3 that $\mathbf{c}$ is $(5 \delta, R)$ vanishing with $5 \delta<\delta_{0}$ and hence also $\left(\delta_{0}, R\right)$-vanishing. According to Lemma 5.2, we have that $|\mathbf{G}|^{p} \in L^{q, \varphi}(\Omega)$ hence by the Theorem 3.1 $|D u|^{p} \in L^{q, \varphi}(\Omega)$ and the following estimate holds

$$
\left\||D u|^{p}\right\|_{L^{q, \varphi}(\Omega)} \leq c\left\||\mathbf{G}|^{p}\right\|_{L^{q, \varphi}(\Omega)} \leq c\left(\left\||\mathbf{F}|^{p}\right\|_{L^{q, \varphi}(\Omega)}+1\right) .
$$

\section{REFERENCES}

[1] V. Barbu, Nonlinear Semigroups and Differential Equations in Banach Spaces. Editura Academiei Republicii Socialiste Romania, Bucharest; Noordhoff International Publishing, Leiden (1976).

[2] S.-S. Byun, Optimal $W^{1, p}$ regularity theory for parabolic equations in divergence form, $J$. Evol. Equ., 7, No. 3 (2007), 415-428.

[3] S.-S. Byun, J. Oh, L. Wang, Global Calderón-Zygmund theory for asymptotically regular nonlinear elliptic and parabolic equations, Intern. Math. Res. Not., 2015, No- 17 (2015), 8289-8308.

[4] S.-S. Byun, D.K. Palagachev, Weighted $L^{p}$-estimates for elliptic equations with measurable coefficients in nonsmooth domains, Pot. Anal., 41, No. 1 (2014), 51-79.

[5] S.-S. Byun, D.K. Palagachev, Morrey regularity of solutions to quasilinear elliptic equations over Reifenberg flat domains, Calc. Var. Part. Differ. Equ., 49, No. 1-2 (2014), 37-76.

[6] S.-S. Byun, D.K. Palagachev, L. Softova, Global gradient estimates in weighted Lebesgue spaces for parabolic operators, Annal. Acad. Scient. Fenn., Series A I. Mathematica, 41, No. 1 (2016), 67-83.

[7] S.-S. Byun, S. Ryu, Global weighted estimates for the gradient of solutions to nonlinear elliptic equations, Ann. Inst. Henri Poincaré, Anal. Non Linéaire, 30 (2), (2013), 291-313.

[8] S.-S.Byun, L. Softova, Gradient estimates in generalized Morrey spaces for parabolic operators, Math. Nachr., 288, No. 14-15 (2015), 1602-1614.

[9] S.-S. Byun, L. Wang, Elliptic equations with BMO coefficients in Reifenberg domains, Comm. Pure Appl. Math., 57, No. 10 (2004), 283-310.

[10] S.-S. Byun, L. Wang, Elliptic equations with BMO nonlinearity in Reifenberg domains, Adv. Math., 219, No. 6 (2008), 1937-1971.

[11] S. Campanato, Proprietà di Hölderianità di alcune classi di funzioni. Ann. Scuola Norm. Super. Pisa, 17 (1963), 175-188. 
[12] M. Chipot, L.C. Evans, Linearisation at infinity and Lipschitz estimates for certain problems in the calculus of variations, Proc. Royal Soc. Edinb., Section A, 102, No. 3-4 (1986), 291303.

[13] A. Cianchi, L. Pick, Sobolev embeddings into spaces of Campanato, Morrey, and Hölder type, J. Math. Anal. Appl., 282, No.1 (2003), 128-150.

[14] E. DiBenedetto, $C^{1+\alpha}$ local regularity of weak solutions of degenerate elliptic equations, Nonlinear Anal., 7 (8) (1983), 827-850.

[15] M. Foss, Global regularity for almost minimizers of nonconvex variational problems, Ann. Math. Pura Appl., (4) 187, No. 2 (2008), 263-321.

[16] D. Gilbarg, N.S. Trudinger, Elliptic Partial Differential Equations of Second Order, 2nd ed. Springer-Verlag, 1983.

[17] C. Kenig, T. Toro, Poisson kernel characterization of Reifenberg flat chord arc domains, Ann. Sci. École Norm. Sup. (4), 36, No. 3 (2003), 323-401.

[18] T. Mengesha, N. Phuc, Weighted and regularity estimates for nonlinear equations on Reifenberg flat domains, J. Differ. Equ., 250, No. 5 (2011), 2485-2507.

[19] E. Milakis, T. Toro, Divergence form operators in Reifenberg flat domains, Math. Zeitschr., 264 (2010), 15-41.

[20] C.B. Morrey, On the solutions of quasi-linear elliptic partial differential equations, Trans. Amer. Math. Soc., 43 (1938), 126-166.

[21] C.B. Morrey, Second order elliptic equations in several variables and Hölder continuity, Math. Zeitschr., 72 (1959), 146-164.

[22] E. Nakai, Hardy-Littlewood maximal operator, singular integral operators and the Riesz potentials on generalized Morrey spaces, Math. Nachr., 166 (1994), 95-103.

[23] D. Palagachev, L. Softova, The Calderón-Zygmund property for quasilinear divergence form equations over Reifenberg flat domains, Nonlin. Anal., Theory Methods Appl., Ser. A, 74, No. 5 (2011), 1721-1730.

[24] E.R. Reifenberg, Solution of the Plateau problem for $m$-dimensional surfaces of varying topological type, Acta Math., 104 (1960), 1-92.

[25] J.P. Raymond, Lipshitz regularity of solutions to some asymptotically convex problems, Proc. Royal Soc. Edinb., Section A, 117, No. 1-2 (1991), 59-73.

[26] C. Scheven, T. Schmidt, Asymptotically regular problems I: Higher integrability, Journ. Differ. Equ., 248, No. 4 (2010), 745-791.

[27] C. Scheven, T. Schmidt, Asymptotically regular problems II: Partial Lipschitz continuity and a singular set of positive measure, Ann. Scuola Norm. Super., (5) 8, No. 3 (2009), 469-507.

[28] A. Torchinsky, Real Variable Methods in Harmonic Analysis, Pure and Applied Mathematics, 123. Academic Press, Inc., Orlando, FL, 1986.

[29] T. Toro, Doubling and flatness: geometry of measures, Notices Amer. Math. Soc., 44, No. 9 (1997), 1087-1094. 